\title{
Piezoelectric Energy Harvester for a CMOS Wireless Sensor
}

\author{
Nuno Mancelos ${ }^{2}$, Joana Correia ${ }^{2}$, Luís Miguel Pires ${ }^{1,2}$, Luís B. Oliveira ${ }^{1,2}$, \\ and João P. Oliveira ${ }^{1,2}$ \\ ${ }^{1}$ Centre for Technologies and Systems (CTS) - UNINOVA \\ ${ }^{2}$ Dept. of Electrical Engineering (DEE), Universidade Nova de Lisboa (UNL) \\ Campus FCT/UNL, 2829-516, Caparica, Portugal \\ \{n.mancelos, jm.correia, 1.pires\}@campus.fct.unl.pt, \\ jpao@fct.unl.pt
}

\begin{abstract}
The emerging of collective awareness platforms opened a new range of driven forces that will converge to more sustainable systems. To achieve this task, these platforms have to support an increasing number of more sophisticated remote sensors and actuators that will need to cooperate smartly and strongly with each other in a mesh type of intelligent interconnectivity. These remote smart miniaturized nodes can add noninvasive intelligence but suffer from lifetime performance due to the small quantity of energy available in micro batteries. Therefore, harvesting energy from the environment is a promising technique. This work presents the study and experimental evaluation of a flexible piezoelectric material to validate the use of a piezoelectric harvester in a CMOS wireless actuator/sensor node.
\end{abstract}

Keywords: Energy harvesting, Piezoelectric transducers, Self-powered, Microsystems, Smart systems, Wireless sensor nodes, MEMS, WSN.

\section{$1 \quad$ Introduction}

The recent advances in ultra-low-power device integration, communication electronics and Micro Electro-Mechanical Systems (MEMS) technology have fuelled the emerging technology of Wireless Sensor Networks (WSNs). The spatial distributed nature of WSNs often requires batteries to power the individual sensor nodes. One of the major limitations on performance and lifetime of WSNs is the limited capacity of these finite power sources, which must be manually replaced when they are depleted. Moreover, the embedded nature of some of the sensors and hazardous sensing environment make battery replacement very difficult and costly. The process of harnessing and converting ambient energy sources into usable electrical energy is called energy harvesting. Energy harvesting raises the possibility of self-powered systems, which are ubiquitous and truly autonomous, and without human intervention for energy replenishment. Among the ambient energy sources such as solar energy, heat, and wind, mechanical vibrations are an attractive ambient source mainly because they are widely available and are ideal for the use of 
piezoelectric materials, which have the ability to convert mechanical strain energy into electrical energy.

Contributions of this paper are summarized as follows. Section 2 presents the relationship to Collective Awareness Systems. In Section 3, state of the art of energy harvesting transducers is presented. In Section 4 the concept of synergy between piezoelectric energy harvesters and WSN is justified and a block diagram of the proposed self-powered Wireless Actuator/Sensor Node is presented. Section 5 presents experimental evaluation of the piezoelectric harvester. Finally some conclusions are drawn in section 6.

\section{Relationship to Collective Awareness Systems}

Nowadays, the emergence of collective awareness systems is pushing the performance of end user interactive objects. The support framework based in a interconnected objects and things (Internet of Things, IoT) is the bridge that combines technologies and components from micro-systems (miniaturized electric, mechanical, optical and fluid devices) with knowledge, technology and functionality from several areas of research.

However, Harbor Research [1] defines smart systems as a new generation of systems architecture (hardware, software, network technologies, and manage services) that provides real-time awareness based on inputs from machines, people, video streams, maps, new feeds, sensors and more that integrate people, process, and knowledge to enable collective awareness and decision making.

WSN provide endless opportunities, but at the same time pose formidable challenges, such as the fact that energy is a scarce and usually non-renewable resource. However, as part of WSN, micro-systems could provide advances in low power Very Large Scale Integration (VLSI), embedded computing, communication hardware, and in general, the convergence of computing and communications, are making this emerging technology a reality. Likewise, advances in nanotechnology and MEMS are pushing toward networks of tiny distributed sensors and actuators.

As mentioned in the previous section, energy harvesting can dramatically extend the operating lifetime of nodes on WSN. Finally, this technology enables battery less operation and reduces the operation costs of WSN, which are mainly due to battery replacement.

\section{Energy Harvesting from the Surroundings}

Energy harvesting techniques developed for micropower generators deal with the challenge of scavenging and making use of residual energy present in ambient sources, usually energy in the form of light, Radio Frequency (RF) electromagnetic radiation, thermal gradients and many sources of motion, namely rotation, vibration and fluid flow. Energy harvesting transducers that make use of energy in the form of motion are called Electromechanical and are separated in three different groups: electromagnetic, electrostatic and piezoelectric transducers. Piezoelectric transducers 
make use of the piezoelectric effect, which refers to the accumulation of an electrical charge in some solid materials, like crystals and certain ceramics, when a mechanical stress is applied to them. The effect is reversible, which means that movement, in the form of oscillation, can occur for the resonance frequency of the particular piezoelectric material to which an electrical charge is applied. These transducers are usually designed to harvest energy from vibration sources. Their harvesting optimization highly depends on the success of the characterization of the vibration from which the energy is to be harvested.

The three electromechanical transducer types are very different from each other. In general, power efficiency of a mechanical transducer could be considered as the ratio between the electrical power it delivers and the mechanical power it receives from the motion source. However, comparing different transducers is not trivial.

In [2], a variant of harvester effectiveness performance indicator is introduced, the Volume Figure of Merit (VFM), which is used to compare the performances of energy harvesting electromechanical transducers as a function of their size. The devices chosen for the actual comparison are electromechanical transducers of the three types mentioned above. Table 1 contains the two best results achieved for each of the transducer types. The main conclusion taken from the presented results is that piezoelectric transducers achieve reasonable values for power efficiency, when compared to the other electromechanical energy harvesting transducers.

Table 1. VFM for the Three Transducers Types (Extracted from [2])

\begin{tabular}{ccc}
\hline Transducer type & Reference & VFM[\%] \\
\hline \multirow{2}{*}{ Electromagnetic } & {$[3]$} & 0.52 \\
& {$[4]$} & 0.64 \\
Electrostatic & {$[5]$} & 0.06 \\
\multirow{2}{*}{ Piezoelectric } & {$[6]$} & 0.68 \\
& {$[7]$} & 1.39 \\
& {$[8]$} & 1.74 \\
\hline
\end{tabular}

\section{Synergy between Piezoelectric Energy Harvesters and Wireless Sensor Networks}

Wireless Actuator/Sensor Nodes represent a wide range of devices with different functionalities and characteristics with an estimated power consumption level that can be retrieved from the data available in [9]. Some commercially available actuator sensor nodes, namely Crossbow MICAZ, Intel Mote 2 and Jennie JN5139, have power consumption levels of $2.8 \mathrm{~mW}, 12 \mathrm{~mW}$ and $3 \mathrm{~mW}$, respectively.

In [10], the power consumption of a wireless sensor node based on the Nordic RF24L01 wireless transceiver is analysed. The conclusion reached is that the RF transceiver is responsible for $74 \%$ of the power consumption, the rest being associated with the power consumptions of the microcontroller, the power management module, sensors, actuators and Analog to Digital Converter (ADCs).

The power supplied by a piezoelectric energy harvester depends greatly on the piezoelectric transducer's characteristics and the vibration conditions it is submitted to. Furthermore, for each piezoelectric transducer setup there are optimal vibration 
conditions, which should match the resonance frequency of the piezoelectric material, and its optimal acceleration, which is directly related to the vibration amplitude. Considering an optimized energy harvesting setup regarding the transducer and the characterisation of the vibration frequency, the circuit responsible for rectification and eventual voltage supply regulation must be designed and implemented. It should be noted that this circuit has a power efficiency associated to it. As an optional component in the power management part of the system, and depending, once again, on the vibration predictability, an energy storage device might be considered.

Two examples of piezoelectric energy harvesters implemented with optimal vibration characteristics and resistive loads that maximize the power output are given in [9] and [11]. In the first case, a power output of $3 \mathrm{~mW}$ was achieved, while in the second work a value of $4.4 \mathrm{~mW}$ was also experimentally verified. For the first implementation, a Volture V22BL piezoelectric transducer was used, with vibration characteristics of $50 \mathrm{~Hz}$ and $1 \mathrm{~g}$. The second example makes use of a Piezo Systems T226-A4-503X piezoelectric transducer, with vibration characteristics of $50 \mathrm{~Hz}$ and $0.5 \mathrm{~g}$. It should be noted that the power outputs are achieved using optimized resistive loads for each of the situations.

Although power consumption and power output levels do not match, energy harvesting solutions can be made possible using a duty cycling technique, making use of a battery or a supercapacitor. This technique consists in switching periodically the sensor node on and off. Since the wireless sensor node doesn't have to be constantly communicating, the energy harvested by the transducer is used in the communication time interval of the sensor node and stored in the battery (or capacitor) during the rest of the cycle (switched off time interval). In [2], a duty cycle of $1.6 \%$ is used for a solution using a piezoelectric energy harvester powering a custom designed radio transceiver that requires $12 \mathrm{~mW}$ when transmitting. The power consumption values previously presented, from [9], are based in operating conditions of $1 \%$ communication, $10 \%$ processing and $89 \%$ sleeping. The proposed block diagram of a self-powered actuator/sensor node is presented in Fig. 1.

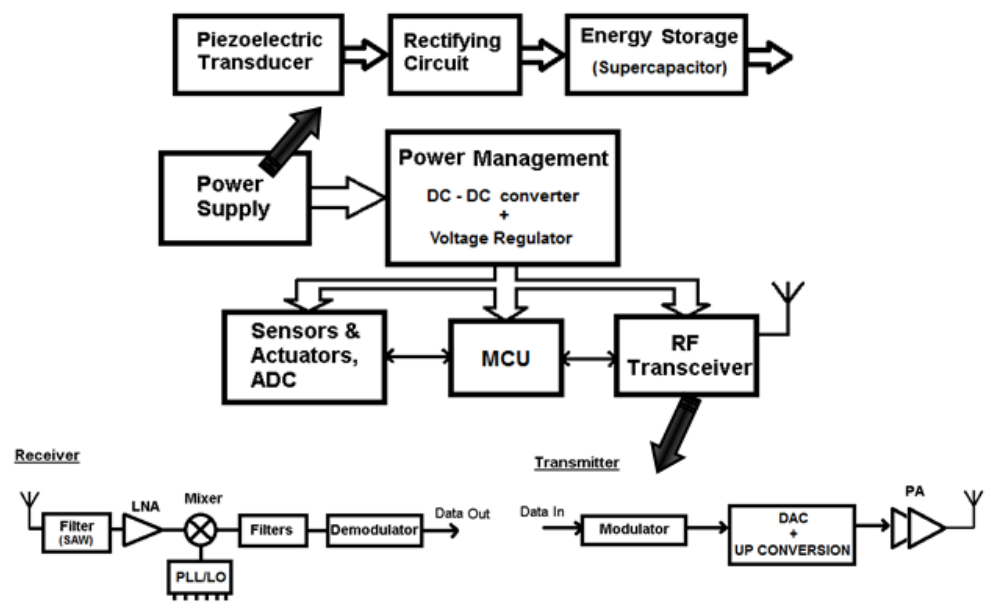

Fig. 1. Block diagram of a Piezoelectric-powered WSN 


\section{Experimental Evaluation of the Piezoelectric Energy Harvester}

The chosen piezoelectric energy harvester Midé Volture ${ }^{\mathrm{TM}}$ V21bl [12], designed for vibration energy harvesting, is presented in Fig. 2. It uses the piezoelectric characteristics of its specific piezoceramic material to produce electrical charge when mechanically stimulated [13] and consists in a package of piezoelectric materials in a protective skin, with four pre-attached electrical leads.
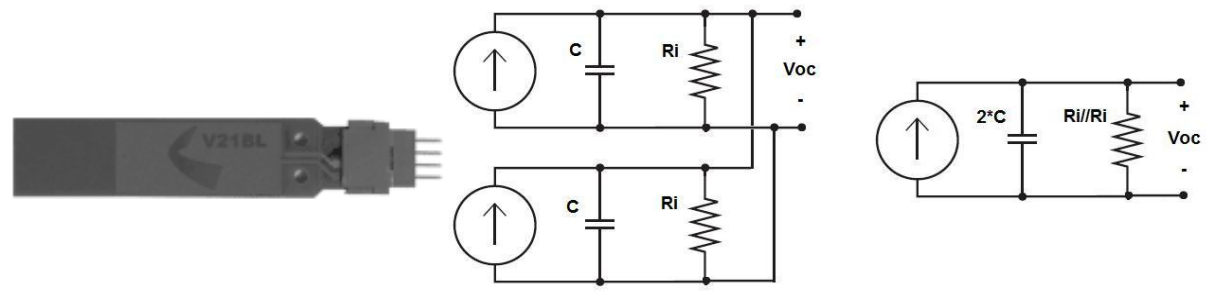

Fig. 2. Midé Volture ${ }^{\mathrm{TM}}$ V21bl [12]

Fig. 3. Parallel equivalent circuit

The piezoelectric harvester includes two electrically isolated piezo wafers, which may be used independently or in one of two possible combinations. These combinations can be optimised for increased output voltage (series configuration) or increased output current (parallel configuration). For this implementation, and since the desired output voltage is relatively low compared to the range of typical output voltages of the piezoelectric harvester, the parallel configuration is used, in order to maximize the output current needed to charge the capacitor. Fig. 3 presents the final equivalent circuit of the parallel configuration of the two wafers.

A block diagram of the test setup is shown in Fig. 4, which comprises three complementary parts:

1. a test vibration module which is formed by an audio amplifier (LM386) and vibration speaker coupled to the piezoelectric transducer;

2. a rectifying diode bridge plus a fast charge capacitor;

3. a data-acquisition subsystem, consisting of a 10-bit ADC and a Microcontroller (MCU) connected to both a Liquid Crystal Display (LCD) Module (HD44780 module, 16X2 LCD Panel) and a USB connection to a computer.

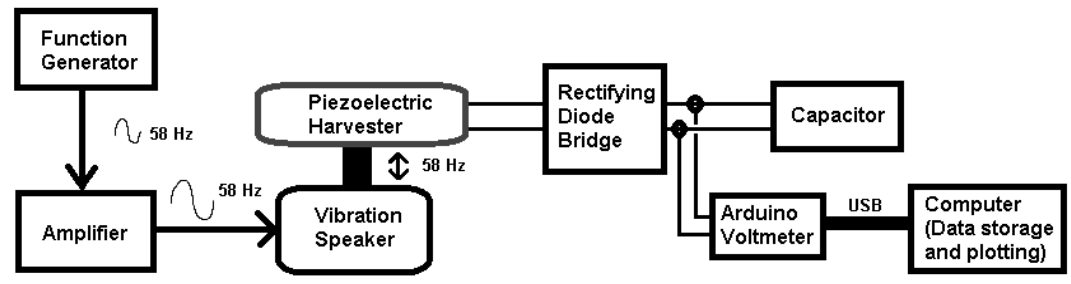

Fig. 4. Block diagram of the test setup 
A picture taken of the test setup is shown in Figure 5.

The energy stored in the capacitor is calculated using the standard equation given by,

$$
\mathrm{E}=\left(\frac{1}{2}\right) \mathrm{cV}^{2}
$$

For the Energy calculation, the actual capacitance of the parallel of capacitors was set to $2100 \mu \mathrm{F}$. In fact, this energy was calculated using the discharge time of the capacitor (from $1.3 \mathrm{~V}$ to $0 \mathrm{~V}$ ), which is approximately 21 seconds, Fig. 6 a). Using this discharge time, the resistance value of the RC circuit $(2 \mathrm{~K} \Omega$ ) and the RC time constant approximation, the actual capacitance of the capacitor can be calculated. Using this value, the estimated stored energy in the capacitor is $1.77 \mathrm{~mJ}$, for $1.3 \mathrm{~V}$.

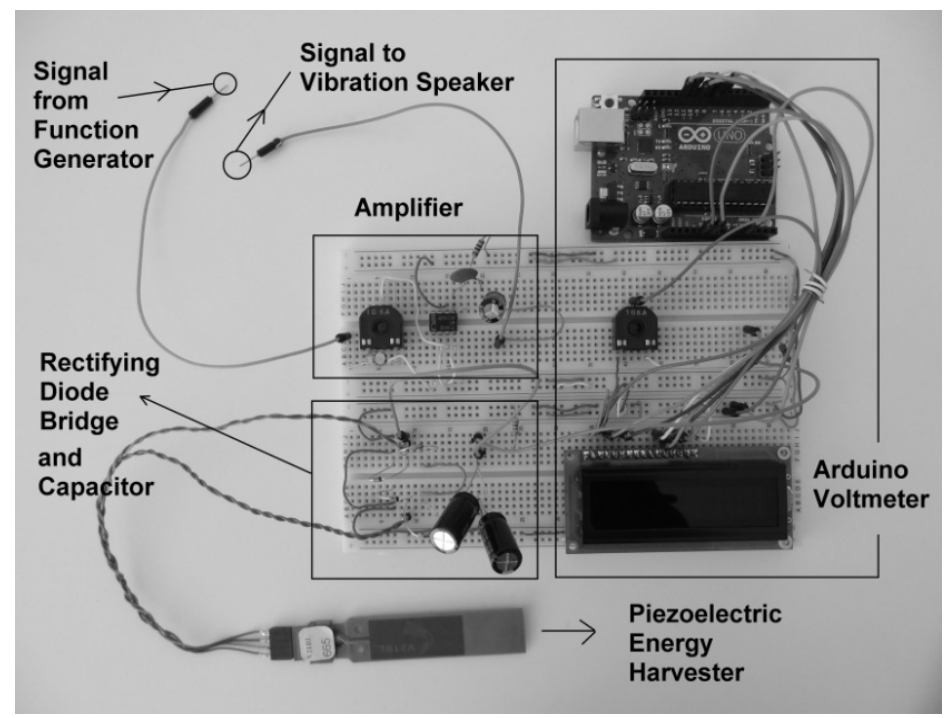

Fig. 5. Photograph of the implementation

Two different methods of vibration were used during the tests: the manually induced and the one produced by the small amplitude vibration speaker. The optimal vibration frequency was found for the second case.

For the manually induced movement test, a constant oscillation frequency was emulated. Given the equivalent circuit of the piezoelectric harvester, previously presented in Fig. 3, the expected behaviour of the charging circuit is described by,

$$
V_{C}=V_{\text {Final }} *\left(1-\mathrm{e}^{-\frac{\mathrm{t}}{\mathrm{RC}}}\right)
$$

where $V_{C}$ is the instant voltage across the capacitor while $V_{\text {Final }}$ depends on the open circuit voltage of the piezoelectric harvester. The charging time was approximately 68 seconds, Fig. 6 b). After some testing using a speaker, the optimal vibration frequency for energy harvesting purposes was found to be $58 \mathrm{~Hz}$. Three tests were run with this 
vibration frequency, following the same procedure. The charging process was interrupted when the capacitor reached $1.3 \mathrm{~V}$, after which the capacitor is connected to a $2 \mathrm{k} \Omega$ resistor. Fig. 6 a) shows the results of these three tests.
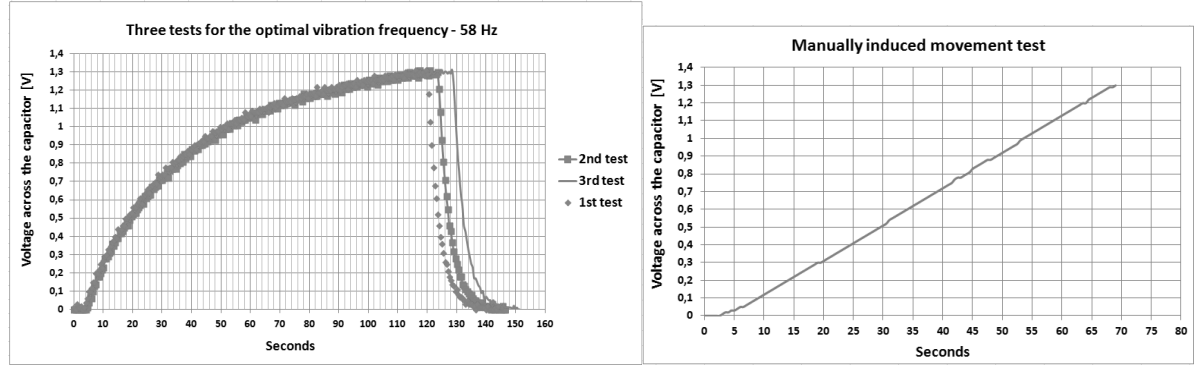

Fig. 6. a) Three tests for the optimal vibration frequency - $58 \mathrm{~Hz}$; b) manually induced movement test

The charging time of the capacitor (from $0 \mathrm{~V}$ to $1.3 \mathrm{~V}$ ) is approximately 125 seconds (medium value). It is greater than the one achieved with the first test (the manually induced movement test), proving that the tip-to-tip displacement amplitude, related to the vibration acceleration, is an important factor in the energy harvesting implementation, using this particular piezoelectric energy harvester.

Using non-optimal vibration frequencies for the vibration speaker case, the time the piezoelectric harvester takes to charge the capacitor up to the same voltage level is greater. To prove this, two non-optimal frequencies were chosen: $60 \mathrm{~Hz}$, which is above the optimal frequency, and $56 \mathrm{~Hz}$, which is below. The charging times for these tests are approximately 250 seconds for the first one and 180 seconds for the second. Both of them are greater than the one achieved for the optimal vibration frequency, 125 seconds. Table 2 shows practical results of this work.

Table 2. Summary of the experimental results

\begin{tabular}{ccccc}
\hline Vibration type & $\begin{array}{c}\text { Vibration } \\
\text { frequency }\end{array}$ & $\begin{array}{c}\text { Charging } \\
\text { time (from } \\
\text { 0V to 1.3V) }\end{array}$ & $\begin{array}{c}\text { Energy stored in } \\
\text { the capacitor } \\
\text { after charging } \\
\text { time }\end{array}$ & Used capacitor \\
\hline \multirow{2}{*}{ Vibration Speaker } & $58 \mathrm{~Hz}$ & $125 \mathrm{~s}$ & & $2100 \mu \mathrm{F}$ \\
Manual & $56 \mathrm{~Hz}$ & $180 \mathrm{~s}$ & $1.77 \mathrm{~mJ}$ & \\
\hline
\end{tabular}

\section{Conclusions}

The main conclusion of the present work is that it is possible to implement an energy harvesting solution using the piezoelectric energy harvester Midé Volture ${ }^{\mathrm{TM}} \mathrm{V} 21 \mathrm{bl}$ for low voltage applications, even if the vibration conditions applied to the harvester are not optimal. Comparing the vibration speaker case and the test involving manually induced oscillation, it is possible to conclude that the tip-to-tip displacement 
amplitude is a very important factor in terms of energy generated by the piezoelectric device, more so than the actual selection of the right frequency for the oscillation.

For each configuration of the mechanical vibration method, there is an optimal vibration frequency, which is $58 \mathrm{~Hz}$, in the described case.

Applying this study to a microsensor node makes energy-harvesting operation a possibility for microsensor networks [14] based on CMOS technology.

\section{References}

1. Harbor Research: Machine-to-Machine (M2M) an Smart Systems Forecast 2010-2014, Harbor Research (2010)

2. Mitcheson, P.D., Yeatman, E.M., Rao, G.K., Holmes, A.S., Green, T.C.: Energy Harvesting From Human and Machine Motion for Wireless Electronic Devices. In: Mitcheson, P.D. (ed.), vol. 96(9) (September 2008)

3. Ching, N.N.H., Wong, H.Y., Li, W.J., Leong, P.H.W., Wen, Z.: A laser-micromachined vibrational to electrical power transducer for wireless sensing systems. In: Proc. 11th Int. Conf. Solid-State Sensors Actuators, Munich, Germany (June 2001)

4. Ching, N.N.H., Wong, H.Y., Li, W.J., Leong, P.H.W., Wen, Z.: A laser-micromachined multi-modal resonating power transducer for wireless sensing systems. Sensors Actuators A, Phys. 97-98, 685-690 (2002)

5. Despesse, G., Chaillout, J., Jager, T., Léger, J.M., Vassilev, A., Basrour, S., Charlot, B.: High damping electrostatic system for vibration energy scavenging. In: Proc. 2005 Joint Conf. Smart Objects Ambient Intell. - Innov. Context-Aware Serv., Grenoble, France, pp. 283-286 (2005)

6. Arakawa, Y., Suzuki, Y., Kasagi, N.: Micro seismic electrets generator using electrets polymer film. In: Proc. 4th Int. Workshop Micro and Nanotechnology for Power Generation and Energy Conversion Applicat., Kyoto, Japan, pp. 187-190 (November 2004)

7. Fang, H.B., Liu, J.Q., Xu, Z.Y., Dong, L., Wang, L., Chen, D., Bing-Chu, C., Liu, Y.: Fabrication and performance of MEMS-based piezoelectric power generator for vibration energy harvesting. J. Microelectron 37(11), 1280-1284 (2006)

8. Roundy, S., Wright, P.K., Rabaey, J.M.: Energyscavenging for wireless sensor networks, 1st edn. Kluwer Academic, Boston (2003)

9. Yoon, Y.-J., Park, W.-T., Li, K.H.H., Ng, Y.Q., Song, Y.: A Study of Piezoelectric Harvesters for Low-Level Vibrations in Wireless Sensor Network. International Journal of Precision Engineering and Manufacturing 14(7) (July 2013)

10. Huang, L., Pop, V., Francisco, R.d., Vullers, R., Dolmans, G., de Groot, H., Imamura, K.: Ultra Low Power Wireless and Energy Harvesting Technologies - An Ideal Combination. In: IEEE, Eindhoven, The Netherlands and Osaka, Japan (2010)

11. Zhou, D., Kong, N., Ha, D.S., Inman, D.J.: A Self-powered Wireless Sensor Node for Structural Health Monitoring. In: Health Monitoring of Structural and Biological Systems, USA (2010)

12. Datasheet: Midé: PIEZOELECTRIC ENERGY HARVESTERS. Volture ${ }^{\mathrm{TM}}$

13. Datasheet: Volture Products - Material Properties. Volture Products - Material Properties, Volture $^{\mathrm{TM}}$

14. Calhoun, B.H., Daly, D.C., Verma, N., Finchelstein, D., Wentzloff, D., Wang, A., Cho, S.H., Chandrakasan, A.: Design considerations for ultra-low energy wireless microsensor nodes. IEEE Trans. Comput. 54(6), 727-740 (2005) 